\title{
Analysis of Clinical Symptoms Improvement in Treatment of Severe Pneumonia Caused by Multi- drug Resistant Bacterial Infection by Bronchoscopy Alveolar Lavage
}

\author{
Xiang Wang, Zuozhou Xie, Jinhong Zhao, Yi Liu* \\ Kunming Second People's Hospital, Kunming 650203, Yunnan Province, China \\ *Corresponding author: Yi Liu,wxm12311y@sina.com
}

\begin{abstract}
Objective: To explore the effects of bronchoscopy alveolar lavage in the treatment of severe pneumonia caused by multiple drug bacterial infection. Methods: A total of 84 patients with severe pneumonia infected by multi-drug resistant bacteria were randomly selected from Kunming Second People's Hospital, which is our hospital from January 2019 to December 2020 for this research. They were divided into a reference group and a study group using a digital table method, with 42 cases in each group. The reference group was given the routine treatment, the research group performed bronchoscopy alveolar lavage on this basis to observe the curative effect. Results: Before treatment, there was no significant difference in serum factor indexes and blood gas analysis indexes between the two groups of patients, $\mathrm{P}>0.05$. After treatment, the time of fever, cough, moist rales disappearing and infection control time in the study group were shorter than those in the reference group, $\mathrm{P}<0.05$. Serum tumor necrosis factor $\alpha$ (TNF- $\alpha$ ), interleukin-6 (IL-6) and interleukin- 8 (IL-8) levels were lower than the reference group, and the arterial partial pressure of oxygen ( $\mathrm{PO} 2)$ and oxygen saturation (SO2) were both lower than the reference group, $\mathrm{P}<0.05$. The effective rate of the treatment in the study group was higher than that in the reference group, $\mathrm{P}<0.05$. Conclusion: Bronchoscopic alveolar lavage treatment can effectively improve the clinical symptoms of patients with severe pneumonia caused by multi-drug resistant bacterial infection, and the effects are significant.
\end{abstract}

Keywords: Bronchoscopy alveolar lavage; Multi-drug resistant bacteria; Severe pneumonia; Clinical symptoms

Publication date: July 2021; Online publication: July 31, 2021

\section{Introduction}

Severe pneumonia is a more common critical illness in clinical practice, among them, the type of pneumonia caused by multi-drug resistant bacterial infection is more common. Once infected, the patient's condition develops extremely fast, often showing symptoms such as fever, cough, and moist rales. At the same time, it can also be accompanied by respiratory failure and body organ failure, which poses a great threat to the patient's life and health. Thus, treatment must be carried out as soon as possible to improve the clinical symptoms and protect the health and safety of patients ${ }^{[1]}$. In the past, conventional drugs were commonly used for treatment, but the desired effect could not be achieved. According to related studies, it was found that the application of bronchoscopy alveolar lavage has an important role in improving the condition. On that basis, this time we will explore the effect of bronchoscopy alveolar lavage on patients with pneumonia caused by multi-drug resistant bacterial infection. 


\section{Materials and methods}

\subsection{Basic information}

A total of 84 patients with severe pneumonia, infected by multi-drug resistant bacteria were randomly selected as research subjects in our hospital from January 2019 to December 2020.

\subsubsection{Inclusion criteria}

(1) All patients were confirmed to meet the criteria for severe pneumonia after examination and were all multi-drug resistant bacteria infection.

(2) Research with informed consent.

\subsubsection{Exclusion criteria}

(1) Patients with severe cardiopulmonary, liver and kidney dysfunction.

(2) Patients with coagulation dysfunction or systemic infection.

(3) Patients with mental illness and cognitive dysfunction.

(4) Patients who are allergic to drugs or cannot tolerate treatment.

Use the digital table method to divide into reference group and study group. Each group has 42 cases. The male and female ratio in the reference group is 24:18. The age limit is 48-64 years old, with an average of $(56.15 \pm 2.36)$ years old. APACHE II score $(16.07 \pm 2.45)$ points. The proportion of male and female in the study group are 25:17, the age limit is 47-65 years old, the average is $(56.24 \pm 2.41)$ years old, and the APACHE II score is $(16.14 \pm 2.37)$ points. The patient data was calculated using statistical software and there was no significant difference $(\mathrm{P}>0.05)$.

\subsection{Method}

The reference group was given the conventional treatment. Firstly, the patients were given oxygen inhalation intervention, and after the venous channel was opened, the patient's water and electrolyte status was reasonably adjusted. Then the water and electrolyte balance was maintained, and nutritional support was given, as well as antibiotics were used to prevent any infection.

The research group performed bronchoscopy alveolar lavage treatment on the basis of the above. Before treatment, the patient needed to be given a high-concentration of oxygen inhalation for about three minutes, and the patient was given midazolam drug sedation, while giving the patient a $2 \%$ concentration of lidoca. Due to organ mucosal anesthesia, negative pressure axis sputum removal intervention was carried out. If the patient has a large amount of sputum, it needed to be repeatedly lavaged with normal saline, the total amount is set to $100 \mathrm{ml}, 2-3$ times a day.

\subsection{Observation indicators}

Observed and counted the time of cough resolution, fever resolution, lung rales resolution, and infection control time of the two groups of patients. The evaluation of treatment effect was that after treatment, all symptoms of the patient have basically subsided, and the absorption range of the lesion was found to be more than $70 \%$ under chest imaging examination, and there was no obvious discomfort, thus the curative effect was significant. If all clinical symptoms have been significantly improved, with a chest imaging it was found that the absorption range of the lesion was within $20-70 \%$ under the academic examination, thus the curative effect was effective. If the symptoms and lesions have not been effectively improved, it would have been invalid. The total effective rate $=($ markedly effective + effective $) /$ total number of cases $\times 100 \%$ [2].

Observed and counted the blood gas analysis indexes and serum inflammatory factors indexes of the two groups of patients before and after treatment. The blood gas analysis indexes arterial partial pressure of oxygen (PO2) and oxygen saturation (SO2) were detected by a blood gas analyzer. Serum inflammatory factors were measured with an automatic biochemical analyzer and enzyme-linked immunosorbent assay 
(ELISA) to detect tumor necrosis factor alpha (TNF- a ), interleukin-6 (IL-6) and interleukin-8 (IL-8) levels.

\subsection{Statistical analysis}

Using SPSS23.0 analysis and processing, the $t$ and $\mathrm{X}^{2}$ tests were performed, expressed as $(\overline{\mathrm{x}} \pm \mathrm{s})$ and $(\mathrm{n} / \%)$. If $\mathrm{P}<0.05$, it means that there is a difference in the data.

\section{Results}

\subsection{Improvement of blood gas analysis indicators}

The test statistics are shown in Table 1. There was no significant difference in blood gas analysis indexes between the two groups before treatment, $\mathrm{P}>0.05$. After treatment, $\mathrm{PO} 2$ and $\mathrm{SO} 2$ in the study group were higher than those in the reference group, $\mathrm{P}<0.05$.

Table 1. Statistical blood gas analysis indicators $(\overline{\mathrm{x}} \pm \mathrm{s})$

\begin{tabular}{cccccc}
\hline \multirow{2}{*}{ Group } & $\mathbf{n}$ & \multicolumn{2}{c}{ PO2 $(\mathbf{m m H g})$} & \multicolumn{2}{c}{ SO2 (\%) } \\
\cline { 3 - 5 } & & Before treatment & Arter treatment & Before treatment & Arter treatment \\
\hline $\begin{array}{c}\text { Reference } \\
\text { group }\end{array}$ & 42 & $56.35 \pm 5.84$ & $64.85 \pm 7.05$ & $90.24 \pm 3.45$ & $93.24 \pm 2.71$ \\
$\begin{array}{c}\text { Research } \\
\text { group }\end{array}$ & 42 & $56.17 \pm 5.91$ & $70.26 \pm 7.15$ & $90.31 \pm 3.51$ & $95.17 \pm 1.98$ \\
t & & 0.1404 & 3.4917 & 0.0922 & 3.7267 \\
P & 0.8887 & 0.0008 & 0.9268 & 0.0004 \\
\hline
\end{tabular}

\subsection{Symptom improvement}

According to the statistics in Table 2., the study group's fever subsidence, cough and moist rales subsidence time, and infection control time were shorter than those of the reference group, $\mathrm{P}<0.05$.

Table 2. Statistics of the time to subside clinical symptoms $[(\overline{\mathrm{x}} \pm \mathrm{s}), \mathrm{d}]$

\begin{tabular}{cccccc}
\hline Group & $\mathbf{n}$ & $\begin{array}{c}\text { Fever fading } \\
\text { time }\end{array}$ & $\begin{array}{c}\text { Cough resolution } \\
\text { time }\end{array}$ & $\begin{array}{c}\text { Wet rales fade } \\
\text { time }\end{array}$ & $\begin{array}{c}\text { Infection control } \\
\text { time }\end{array}$ \\
\hline $\begin{array}{c}\text { Reference } \\
\text { group }\end{array}$ & 42 & $8.57 \pm 2.34$ & $12.53 \pm 3.98$ & $14.38 \pm 2.85$ & $15.84 \pm 1.98$ \\
$\begin{array}{c}\text { Research } \\
\text { group }\end{array}$ & 42 & $4.19 \pm 1.85$ & $8.51 \pm 3.04$ & $11.21 \pm 2.26$ & $10.25 \pm 1.66$ \\
t & & 9.5159 & 5.2020 & 5.6481 & 14.0210 \\
P & & 0.0000 & 0.0000 & 0.0000 & 0.0000 \\
\hline
\end{tabular}

\subsection{Improvement of serum inflammatory indicators}

The test statistics are shown in Table 3. there was no significant difference in inflammatory factor indexes between the two groups before treatment, $\mathrm{P}>0.05$. After treatment, the levels of TNF- $\alpha$, IL- 6 , and IL- 8 in the study group were lower than those in the reference group, $\mathrm{P}<0.05$.

\subsection{Clinical efficacy}

The efficacy evaluation statistics show that the effective rate of treatment in the study group is higher than 
that in the reference group, $\mathrm{P}<0.05$.

Table 3. Statistics of serum inflammatory factors $[(\overline{\mathrm{x}} \pm \mathrm{s}), \mathrm{ng} / \mathrm{L}]$

\begin{tabular}{ccccccc}
\hline \multirow{2}{*}{ Group } & \multicolumn{2}{c}{ TNF- $\alpha$} & \multicolumn{2}{c}{ IL-6 } & \multicolumn{2}{c}{ IL-8 } \\
\cline { 2 - 7 } & $\begin{array}{c}\text { Before } \\
\text { treatment }\end{array}$ & $\begin{array}{c}\text { Arter } \\
\text { treatment }\end{array}$ & $\begin{array}{c}\text { Before } \\
\text { treatment }\end{array}$ & $\begin{array}{c}\text { Arter } \\
\text { treatment }\end{array}$ & $\begin{array}{c}\text { Before } \\
\text { treatment }\end{array}$ & $\begin{array}{c}\text { Arter } \\
\text { treatment }\end{array}$ \\
\hline $\begin{array}{c}\text { Reference } \\
\text { group }\end{array}$ & $67.41 \pm 5.39$ & $47.15 \pm 4.13$ & $78.54 \pm 5.16$ & $58.44 \pm 4.05$ & $73.27 \pm 7.61$ & $54.11 \pm 8.34$ \\
$\begin{array}{c}\text { Research } \\
\text { group }\end{array}$ & $67.25 \pm 5.42$ & $38.26 \pm 3.97$ & $78.91 \pm 5.25$ & $48.37 \pm 3.84$ & $73.15 \pm 6.99$ & $43.19 \pm 7.47$ \\
t & 0.1357 & 10.0571 & 0.3257 & 11.6933 & 0.0753 & 6.3208 \\
P & 0.8924 & 0.0000 & 0.7454 & 0.0000 & 0.9402 & 0.0000 \\
\hline
\end{tabular}

Table 4. Statistical evaluation of efficacy $(\mathrm{n} / \%)$

\begin{tabular}{cccccc}
\hline Group & n & Excellent & Valid & Invalid & Total effective rate \\
\hline Reference group & 42 & 15 & 17 & 10 & 76.19 \\
Research group & 42 & 18 & 22 & 2 & 95.23 \\
X $^{2}$ & & & & 6.2222 \\
P & & & & 0.0126 \\
\hline
\end{tabular}

\section{Discussion}

Severe pneumonia caused by multi-drug resistant bacteria is a relatively common severe disease in the current clinical practice. Usually, for patients whom are affected by this disease, will have a large amount of thick sputum in the body, and the body gas exchange will be severely restricted. This would cause them to cough frequently. Symptoms such as sputum expectoration and moist rales can cause great harm to the life and health of patients, thus timely treatment is needed ${ }^{[3]}$.

In the past, conventional treatments, such as antibiotics, were often used in clinical practice. Although they can improve symptoms to a certain extent, they cannot clear the patient's sputum and it is difficult to achieve the desired effect. Therefore, combined bronchoalveolar lavage is required for treatment to rationalize the patient's sputum. Drain and clear, to ensure that the patient's airway is smooth ${ }^{[4]}$.

Bronchoalveolar lavage treatment can accurately determine the patient's specific lesion location through bronchoscopy, absorb sputum, and accurately wash it. This can more effectively remove the sputum in the patient's lungs, as well as promote alveolar ventilation and the lung environment to be significantly improved. When the patient's lung function reasonably improved, it can also effectively control the infection and help the patient recover quickly ${ }^{[5-8]}$.

In summary, the effect of bronchoscope alveolar lavage in the treatment of patients with severe pneumonia infected by multi-drug resistant bacteria is very significant and can effectively improve clinical symptoms 


\section{Funding}

Fund project no.: 2020-SW (reserved) -52, Kunming Health Commission 1000 project reserved candidates: Study on the diagnostic value of BALF solution acer sequencing and culturing in difficult and severe respiratory infections.

\section{Disclosure statement}

The author declares no conflict of interest.

\section{References}

[1] Luo WJ, Wang CM, Hu YF, et al., 2019, Analysis of the Efficacy of Bronchoalveolar Lavage Tanreqing Injection in the Treatment of Multidrug-Resistant Acinetobacter Baumannii Pneumonia in Critically Ill Patients. Chinese Medicine Guide.

[2] Wang Y, 2019, Clinical Analysis of Electronic Bronchoscope Alveolar Lavage in the Treatment of Severe Pulmonary Infection. Digest of World Latest Medical Information (Electronic Edition), 019(077):101-102.

[3] Pang HB, 2020, The Clinical Effect of Bronchoscopy Lavage Combined with Antibiotics in the Treatment of Refractory Pulmonary Multidrug-Resistant Bacteria Infection. Journal of Practical Clinical Medicine, 024(003): 19-21.

[4] Jun XU, Hui LI, Wen L, et al., 2019, Clinical Effect of Fiberoptic Bronchoscopic Sputum Aspiration and Bronchoalveolar Lavage in the Treatment of Pulmonary Infection in Respiratory Intensive Care Unit.

[5] Fang F, Wang YT, Bi LX, et al., 2020, The Etiological Characteristics of Severe Pneumonia in Children with Respiratory Failure and Analysis of the Therapeutic Effect of Bronchoscopy Alveolar Lavage. Chinese Journal of Nosocomial Infection, 30(08): 165-170.

[6] Gao YW, Song JG, Wang DM, 2020, Efficacy and Mechanism Analysis of Electronic Bronchoscope Alveolar Lavage on Children with Severe Pneumonia. Guizhou Medicine, 44(10):43-44.

[7] Zhang J, 2020, Analysis of the Effect of Bronchoscopy Alveolar Lavage Combined with Ambroxol Hydrochloride in the Treatment of Severe Pneumonia in Children. China Journal of Modern Medicine, (1):64-66. volume

[8] Lei LL, Han ZY, Wang Y, 2019, Analysis of the Efficacy of Bronchoalveolar Lavage in the Treatment of Patients with Ventilator-Associated Pneumonia. Internal Medicine, 014(003): 300302,341 . 